\title{
Performance Evaluation of CLS-DFE for MIMO OFDMA System
}

\author{
Siti Maisurah Sulong, Azlina Idris, and Suzi Seroja Sarnin
}

\begin{abstract}
Orthogonal frequency division multiple access (OFDMA) is a multiuser of digital modulation technique used to increase wireless transmission rate. Signal efficiency is distorted due to Inter symbol interference (ISI) that occurs due to the multipath propagation. Cascaded Least Square (CLS) with Decision Feedback Equalizer (DFE) had been used in this research in order to mitigate ISI in OFDMA system. CLS is combination of two adaptive algorithms which are Least Mean Square (LMS) and Recursive Least Square (RLS). This research also justified that combination of space time frequency block code (STFBC) with multiple input multiple output (MIMO) antenna enable to minimize ISI. The simulation results show the improvement of bit error rate (BER) performance after reducing ISI and also prove that STFBC was able to achieve maximum diversity order in the MIMO OFDMA system.
\end{abstract}

Index Terms - Least mean square (LMS), recursive least square (RLS), cascaded least square (CLS), decision feedback equalizer (DFE).

\section{INTRODUCTION}

A combination of multiple input multiple output (MIMO) is an innovation for antenna technology to significantly increase transmission capacity. Nowadays, MIMO is using together with Orthogonal Frequency Division Multiple Access (OFDMA) as a latest technique that combines frequency division multiple access (FDMA) with Orthogonal Frequency Division Multiplexing (OFDM) by subdividing the bandwith into multiple frequency sub-carriers as stated in [1]. OFDMA has been introduced in the IEEE 802.16 wireless for Metropolitan Area Network (MAN) standard to improve transmission rate in wireless communication system. This technique will assign different groups of carriers to different user.

OFDMA need to maintain their orthogonality between each other to avoid signal loss due to the multipath propogation occur during transmission [2]. Each signal that reach receiver are propogating through different paths and the signal will have different delay time. That reason can still affect system performance even though OFDMA can handle multipath interference at the receiver. Besides that, multipath also can can cause inter symbol interference (ISI) in the wireless system.

Previous researchers [3] stated that, ISI is major problem to

Manuscript received February 10, 2016; revised May 16, 2016. This work was supported in part by Institute of Graduate Study UiTM Shah Alam, Malaysia.

The authors are with the Faculty of Electrical Engineering, UiTM Shah Alam, Malaysia (e-mail: sitimaisurah91@ gmail.com.my, azlina831@salam.uitm.edu.my, suzis045@salam.uitm.edu.my). achieve high speed data transmission because it will attenuate signal in the channel. This physical environment causes some symbols to be spread beyond their given time interval. Due to that reason, it can interfere with the following transmitted symbols. Adaptive algorithm will be used with decision feedback equalizer (DFE) to overcome ISI problem. DFE is much better compare to linear equalizer because it has lower noise enhancement while processing the received signal. Cyclic prefix $(\mathrm{CP})$ as a guard interval will be added to help DFE remove interference as long as the $\mathrm{CP}$ at least equal or larger than channel delay spread. Transmitted symbol that is in linear convolution form change to circular convolution form and ISI will completely eliminated from the system [4].

In order to improve more on wireless communication performance, space time frequency (STF) code had been introduced to distribute the element of the orthogonal design both in time and frequency. STF are promising for achieving high bandwith efficiency, latency and low overhead. Before STF is used, space time (ST) code is the first technique that realize the advantage of diversity of multiple antennas [5] but the performance of ST will degrade in fast time-varying channel. After that, space frequency (SF) has been proposed by replacing time domain with frequency domain but system performance degrade in heavily selective channels. Previously, we can see that ST and SF cannnot achieve maximum diversity but after a few years, there are improvement of performance. They can be used in MIMO system with a few changes that need to be considered but the performance is still not as good as STF [6].

Based on the information above, LMS and RLS will be tested in this project with DFE to reduce ISI. LMS is one of the most widely used adaptive algorithms due to its robustness and simplicity while RLS is also one of adaptive algorithm commonly used due to its fast convergence. This research will prove that combination of both algorithms with equalizer have better performance in MIMO OFDMA. It makes the differences with previous work by [7] and [8] because so far there is no exact research relate to the combination of two adaptive algorithms with equalizer using space time frequency block coding technique for MIMO OFDMA.

\section{METHODOLOGY}

To study the performance of MIMO OFDMA, a full system shown in Fig. 1 below.

A full system shown in Fig. 1 is separated into three main parts; Transmitter, channel and receiver. This system use 2-Tx and 2-Rx antennas. A random data stream is generated and modulator will modulate corresponding subcarrier. OFDMA modulation is generated using inverse fast fourier 
transform (IFFT). It will convert frequency domain data into time domain signal while maintaining the orthogonality of subcarriers. STF implementation consists of two encoders for both diversities. Cyclic prefix will act as a guard interval to help DFE operations. From that inter symbol interference (ISI) can be completely eliminated as long as cyclic prefix is longer than maximum spread of the channel.

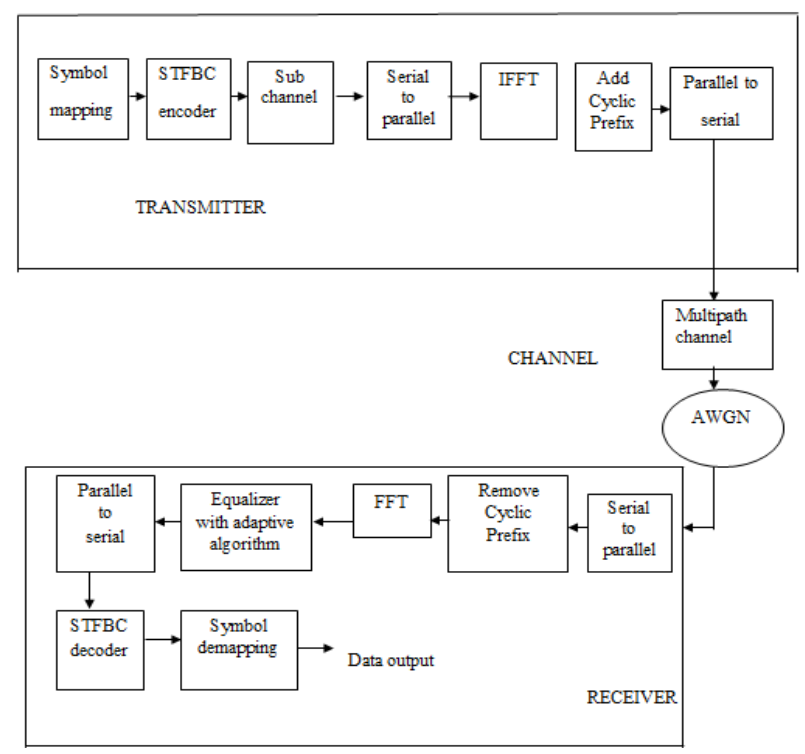

Fig. 1. OFDMA transceiver block diagram.

The channel will simulate like real environment by adding Additive White Gaussian Noise (AWGN) and fading. Cyclic prefix will be removed after reaching receiver end. Receiver performs inverse operation of transmitter. Serial to parallel converter will split OFDMA data from serial to parallel. The role of FFT is to convert time domain to frequency domain and recover the data symbols from orthogonal subcarriers. But still, the output from FFT will contain ISI in it. Equalization technique needs to be used in order to eliminate ISI. This system use DFE as an equalizer. It will minimize the error between actual output and desired output. Basically, it will act as a transversal filter.

Space Time Frequency Block (STFB) coding schemes used to enhance the reliability performance of system. STF implementation consists of two encoders for both diversities where the same symbols are transmitted through multiple antennas at different times and frequency. This analysis use STF coding scheme for CLS and prove that STF is able to achieve maximum diversity order in MIMO OFDMA system.

\section{A. Decision Feedback Equalizer}

Fig. 2 illustrate diagram of DFE and it is slightly different from basic linear equalizer [9].

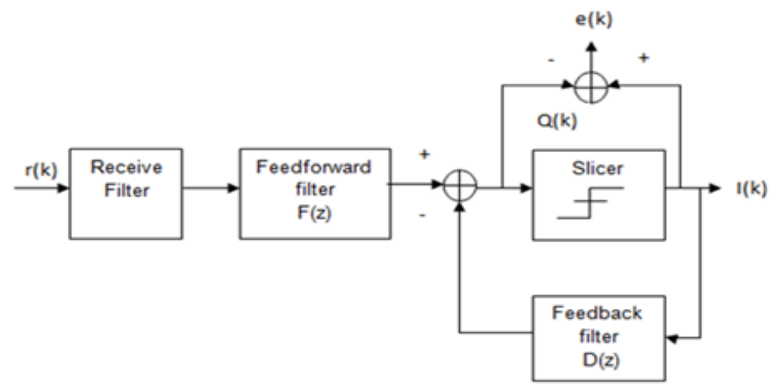

Fig. 2. Decision feedback equalizer block diagram.
This paper is focusing on nonlinear equalizer which is DFE. This equalizer has better performance compare to Linear Equalizer because DFE remove ISI from tails of previously received symbols. Feedback filter (FBF) will eliminate ISI using previous receiver decision while at feed-forward filter (FFF), ICI cancellation occurs on the transmitted symbol. For further research, $F(z)$ and $D(z)$ can be optimized according to any suitable criterion such as Zero Forcing or Minimum Mean Square Error. DFE perform with lower computational complexity and comparable to the behaviour of optimum demodulator. Suitable weighting is required to remove previous symbol values. Slicer will eliminate the noise before feedback to make sure no additional noise enhancement [10]. Error signal, $e(k)$ equation can be written as

$$
e(k)=I(k)-Q(k)
$$

where $I(k)$ is a decision for decision-directed mode and $\mathrm{Q}(\mathrm{k})$ is the decision device input. Updated equation for FFF is;

$$
F(k+1)=F(k)+\mu y[k] e *[k]
$$

While updated equation for FBF is;

$$
D(k+1)=D(k)+\mu k[k] e *[k]
$$

With $y(k)$ as an input at feed forward filter while $z(k)$ is input at feedback filter.

The channel response for this equalizer can be written as

$$
C(z)_{D F E}=c z^{-n o} \div H(z)
$$

While $n_{o}$ is the delay introduce due to the filter operations and $\mathrm{c}$ is a constant gain.

\section{B. Cascaded Least Square}

CLS is a combination of 2 adaptive algorithms that will help DFE to perform well for MIMO OFDMA System. Least Mean Square (LMS) LMS is well known among adaptive algorithm due to its simplicity and low complexity but LMS is not good in terms of convergence speed. In addition, it will not converge if the desired symbols are not correct. The only parameter to be adjusted is the adaptation step size but for this research, only 1 value of step size will be used in the system. The LMS basic equation can be written as stated by [11];

$$
w(k+1)=w(k)+\mu e(k) d(k)
$$

While $\mu$ is the step size, $w(k)$ is filter coefficient vector and $d(k)$ is filter input vector. During each sample period, filter tap weights will be adjusted. Recursive Least Square (RLS) perform better in term of convergence speed compare to LMS. RLS is formalized as below

$$
w(k+1)=w(k)+e(k) \cdot G(k)
$$

Which $e(k)$ is error signal and $G(k)$ is gain vector that can be written as; 


$$
G(k)=P(k) R(k) \div\left[\lambda+R^{T} P(k) R(k)\right]
$$

$P(k)$ is an inverse matrix of input signal while $\lambda$ is forgetting factor that must not be too small in order to avoid algorithm become unstable. Suitable value is between 0 and 1 .

\section{Proposed Algorithm}

This research proposed the signal to enter LMS before entering RLS and equalize by DFE. This is because LMS is simple and easy to perform compare to RLS even though RLS has better performance in terms of convergence speed. The equation can be described as below;

$$
\begin{aligned}
& e_{L M S}=d(n)-w_{L M S}(n) u(n) \\
& y_{n}=w_{L M S}(n) u(n) \\
& e_{R L S}=d(n)-w_{R L S}(n-1) y(n) \\
& z_{C L S}=w_{L M S}(n) u(n) w_{R L S}(n-1) \\
& e_{\text {TOTAL }}=d(n)-z_{C L S}(n)
\end{aligned}
$$

Which $d(n)$ is desired output, $u(n)$ is filter input vector, $y(n)$ is estimated output of LMS. Error for RLS will include $y(n)$ in it. $z_{C L S}$ is final output for CLS before included in DFE operation.

\section{Space Time Frequency}

TABLE I: SPACE TIME FREQUENCY Block Code USING 2 TRANSMIT ANTENNAS AT DIFFERENT FREQUENCY AND TIME

\begin{tabular}{ccc} 
Space Time Frequency & Time 1 & Time 2 \\
\cline { 2 - 3 } & Antenna 1 & Antenna 2 \\
\cline { 2 - 3 } Frequency 1 & $X n$ & $X n+1$ \\
Frequency 2 & $-X n+1^{*}$ & $X n^{*}$ \\
\hline
\end{tabular}

Based on the Table I above, first symbol $X n$ from antenna 1 will be transmitted during time slot 1 and frequency slot 1 followed by $X n+1$ as a second symbol. This type of diversity will distribute element of the orthogonal design in both time and frequency. STF implementation consists of two encoders for both diversities. It will separate spatial and frequency diversity by mapping the information symbols to antenna. Four channels are involved in every time slot whose channel impulse response follows Rayleigh distribution [12]. The received signal will produce

$$
Y=H x+n
$$

With $H$ is for channel influence, $x$ represent signal to be transmitted and $n$ is additive white Gaussian noise. Input signal $x$ that has interference $\mathrm{n}$ is convolved by $H$ in order to produce $Y$.

\section{SimULATION RESULTS}

\section{A. Simulation Parameter}

Based on the Table II, the simulation parameter is referring to IEEE 802.16 protocol. Table III above shows value use to simulate the algorithm and equalizer. The value may change if we want to get more result but need to aware of equalizer stability. Interference is not completely removed from the designated system if the equalizer is unstable.

TABLE II: OFDMA-PHY BASED ON IEEE 8022.16 MOBILE WIMAX PARAMETER

\begin{tabular}{cc}
\hline Parameter & Values \\
\hline FFT size & 1024 \\
Cyclic Prefix & 128 \\
Modulation & QPSK \\
Channel & COST $207 \mathrm{TU}$ \\
Delay & $0.2 \mathrm{e}-60.5 \mathrm{e}-6$ \\
& $1.6 \mathrm{e}-62.3 \mathrm{e}-65.0 \mathrm{e}-6$ \\
Path Gain & 1.122591 .1561 .0591 .0381 .023 \\
\hline
\end{tabular}

\begin{tabular}{ccc}
\multicolumn{2}{c}{ TABLE III: PARAMETER FOR ALGORITHM } \\
\hline Algorithm & Parameter & Value \\
\hline LMS & Step Size & 0.01 \\
RLS & Forget factor & 0.99 \\
DFE & Weight system & 7 \\
\hline
\end{tabular}

\section{B. Diversity Technique}

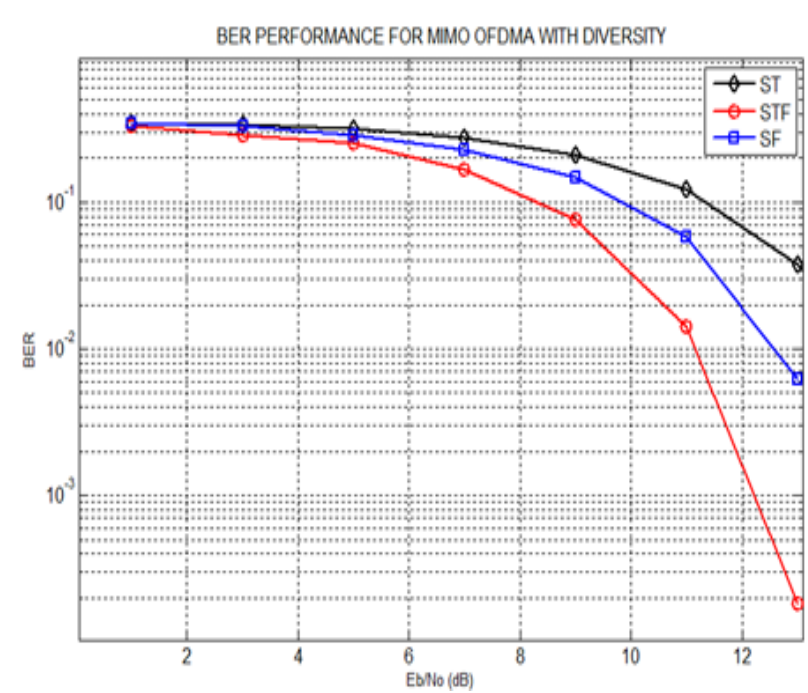

Fig. 3. BER Performance with different type of diversity.

Fig. 3 shows comparison between STF, ST and SF in this system. Diversity gain can be obtained by transmitting signal through spatial, time slot and frequency diversities. As stated in Table I, the signal used all types of diversities and STF proved its efficiency in Fig. 4. ST combiner can be applied after inverse operation of serial to parallel converter at the transmitter but there will be a problem since symbol duration channel will change during the transmission. Performance of ST will degrade in fast time-varying channel. For SF, the performance will degrade in heavily frequency selective channels. SF can be used with low frequency-selectivity in order to make subcarrier spacing very narrow. Due to both ST and SF suffer with their own disadvantage STF had been used in order to relax the requirements for constant channel coefficients in both dimensions. It is proved that STF perform better compare to ST with improvement $28.07 \%$. For SF, STF is still much better with $16.3 \%$ of improvement. Table IV below indicates BER for the diversity. 
TABLE IV: BER FOR DIVERSITY

\begin{tabular}{ccc}
\hline Type & EbNo (dB) & BER \\
\hline Space Time (ST) & 13 & $4 \times 10^{-2}$ \\
Space Frequency (SF) & 13 & $6 \times 10^{-3}$ \\
Space Time Frequency (STF) & 13 & $2 \times 10^{-4}$ \\
\hline
\end{tabular}

\section{Performance of CLS-DFE}

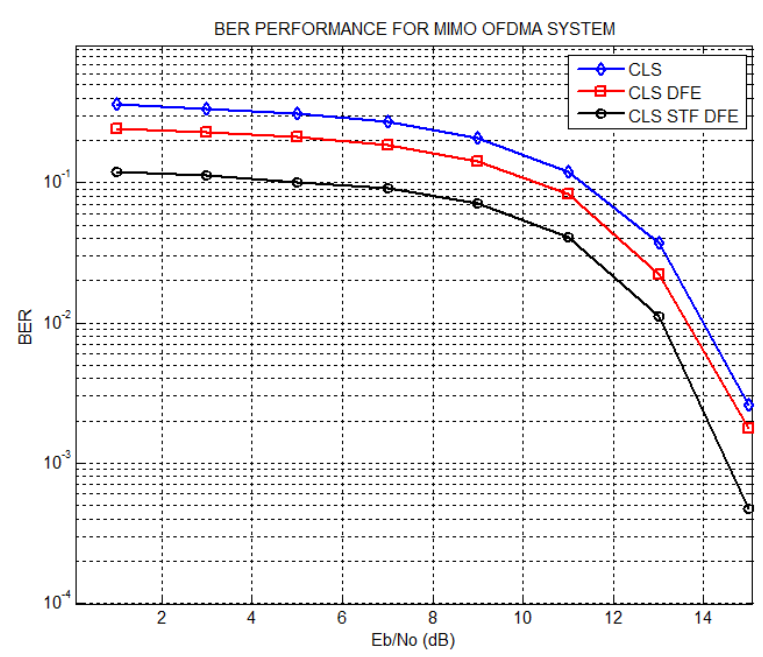

Fig. 4. BER performance with CLS MIMO OFDMA.

Fig. 4 shows performance for CLS in MIMO OFDMA system. The result proved that CLS-DFE with STF perform better by $19.6 \%$ compare to CLS-DFE without diversity. As already stated by other researchers, diversity is important in order to increase reliability of the OFDMA system because each OFDMA consists of subcarrier symbols that need to be transmitted. MIMO is one of the factors that will make sure each symbol reach receiver and not spread away during transmission. CLS STF DFE has 25\% improvement contrast to CLS only because CLS will act as a basic filter without DFE. Besides that, in CLS, LMS has slow convergence speed while RLS has high complexity. Without DFE and STF, this algorithm will not perform well due to their disadvantages. By not using STF, CLS-DFE still has $6.7 \%$ of improvement compare to CLS only. This will explain on how well DFE work to remove ISI in the system because DFE will reduce interference by using previous detector decisions. The system needs to have suitable value of LMS step size and RLS forgetting factor in order to have stable system. For LMS, equalizer become unstable if the step size is too large or too small. RLS has infinite memory length. From (7), when G(k) is increasing, the system become more stable but with slow convergence speed. Equalization error also can be reduced with help from forgetting factor. The combination of 2 algorithms proves it can perform better in MIMO OFDMA system. Table V below stated the BER for the system;

\begin{tabular}{ccc}
\multicolumn{3}{c}{ TABLE V: BER FOR CLS MIMO OFDMA } \\
\hline Type & EbNo $(\mathrm{dB})$ & BER \\
\hline CLS & 13 & $3.7 \times 10^{-2}$ \\
CLS DFE & 13 & $2.2 \times 10^{-2}$ \\
CLS STF DFE & 13 & $1.1 \times 10^{-2}$ \\
\hline
\end{tabular}

\section{ACKNOWLEDGMENT}

The authors wish to thank members of Wireless Communication Technology Group, Faculty Electrical Engineering UiTM Shah Alam, Selangor, Malaysia for the guidance and support during the support.

\section{REFERENCES}

[1] W. Forum, "Mobile WiMAX-part 1: A technical overview and performance evaluation," White Pap. vol. 2, no. 8, p. 15, 2006.

[2] M. Morelli, "Timing and frequency synchronization for the uplink of an OFDMA system," IEEE Trans. Commun., vol. 52, no. 2, pp. 296-306, Feb. 2004.

[3] T.-H. Kim and J.-K. Kang, "A high-speed adaptive linear equalizer with ISI level detection using periodic training pattern," in Proc. 2012 Int. SoC Des. Conf., Nov. 2012, pp. 419-422.

[4] G. L. Stüber, J. R. Barry, S. W. Mclaughlin, S. Member, Y. E. G. Li, M A. N. N. Ingram, and T. G. Pratt, "Broadband MIMO-OFDM wireless communications," Proceedings of the IEEE, vol. 92, no. 2, pp. 271-294, 2004.

[5] H. Bolcskei and A. J. Paulraj, "Space-frequency coded broadband OFDM systems," in Proc. Wireless Communications and Networking Confernce, 2000, no. 650, pp. 1-6.

[6] M. N. Seyman, "MIMO-OFDM channel estimation using ANFIS," Electronics and Electrical Engineering, vol. 120, no. 4, pp. 75-78, 2012.

[7] W. Jian, C. Yu, and L. Wang, "OFDM adaptive digital predistortion method combines RLS and LMS algorithm," in Proc. 2009 4th IEEE Conference on Industrial Electronics and Applications, 2009, pp. 3900-3903.

[8] T. T. N. Do, "Improving performance of wireless communication systems using adaptive space-time scheme," in Proc. International Symposium on Electrical \& Electronics Engineering, 2007, pp. $42-47$.

[9] S. Senol, "Performance comparison of adaptive decision feedback equalizer and blind decision feedback equalizer," The Middle East Technical University, 2004.

[10] A. Idris, S. M. Sulong, N. Ya'acob, and S. S. Sarnin, "Reduction of ISI using different equalization of adaptive algorithm for mobile WiMAX communication system," in Proc. 2014 IEEE Symposium on Wireless Technology and Applications, 2014, pp. 75-80.

[11] P. Sivakumar, K. Rajesh, and M. Rajaram, "A new normalized block LMS based adaptive decision feedback equalizer for wireless communications," in Proc. 2012 International Conference on Advances in Engineering, Science and Management, 2012, no. 1, pp. $116-120$.

[12] A. Idris and K. Dimyati, "Diversity technique for multiple input multiple output orthogonal frequency division multiplexing (MIMO-OFDM) system using a new subcarrier mapping scheme," International Journal of the Physical Sciences, vol. 6, no. 16, pp. 3879-3884, 2011.

Siti Maisurah Sulong received her B.Eng. (Hon.) degree in communication from Universiti Teknologi MARA (UiTM), Shah Alam in 2014. She currently pursues her M.Sc. degree (electrical) in UiTM. Her research interests include wireless telecommunication, OFDMA for the WiMax, adaptive algorithm, equalizers and diversity scheme.

Azlina Idris received her B.Eng. (Hon.) degree from Leeds Metropolitan University and M.Sc. degree from Universiti Teknologi Malaysia. She completed her $\mathrm{PhD}$ degree in Universiti Malaya and now holds position as a senior lecturer in UiTM Shah Alam. Besides that, she is also the coordinator for student employability. Her research interests include pair wise error probability in OFDM, diversity scheme, wireless interference and equalizers.

Suzi Seroja Sarnin received her B.Eng. (Hon) degree from Universiti Teknologi Malaysia (UTM), Malaysia in 1999 and M.Sc. (microelectronic), degree from Universiti Kebangsaan Malaysia (UKM), Malaysia in 2006 respectively. She currently pursues her $\mathrm{PhD}$ degree in UiTM. Her research interests include channel estimation, coding algorithm for CDMA and WCDMA, mobile telecommunication, synchronization algorithm in OFDM for the Wimax technology application. 